\title{
Migración y representaciones regionales: discursos sobre la Región de Antofagasta
}

Luis Miguel Rodrigo. Universidad Católica del Norte, Antofagasta, Chile. Miguel Atienza. Universidad Católica del Norte, Antofagasta, Chile.

RESUMEN | El estudio de la migración interna se ha basado tradicionalmente en una concepción banal del espacio, sin tener en cuenta cómo las representaciones regionales, el espacio construido socialmente, afectan la decisión de migrar a otra región. Este trabajo utiliza las metodologías del grupo de discusión y el análisis sociológico del discurso para identificar los discursos de los futuros profesionales de otras regiones de Chile sobre la Región de Antofagasta, área minera localizada en el norte del país. A pesar de la prosperidad creciente de esta región durante las dos últimas décadas, los discursos que la representan poseen una denotación negativa, con distintos rasgos según la distancia socioespacial a esta región y el género del hablante. Estas representaciones podrían limitar la llegada de migrantes calificados y mujeres a Antofagasta y afectar negativamente el desarrollo futuro de la región.

PALABRAS CLAVE $\mid$ migración, sociología urbana, desarrollo regional y local.

ABSTRACT | Research on internal migration has been traditionally based on a banal conception of space, without taking into account how regional representations, the socially constructed space, affect the decision of migrating to another region. This work uses the methodologies of discussion group and sociological discourse analysis to identify the discourses of future professionals from other regions in Chile about the Region of Antofagasta, a mining area located in Northern Chile. Despite the growing prosperity of this region during the past two decades, the discourses that represent Antofagasta have a negative denotation with different features according to the socio-spatial distance to this region and gender. These representations could reduce the arrival of qualified migrants and women to Antofagasta and negatively affect the future development of the region.

KEY WORDS | migration, urban sociology, regional and local development.

Recibido el 14 de mayo de 2012, aprobado el 5 de diciembre de 2012

E-Mail: Luis Miguel Rodrigo, lrodrigo@ucn.cl | Miguel Atienza, miatien@ucn.cl 


\section{Introducción}

La atracción de trabajadores calificados es ampliamente reconocida como un motor del crecimiento regional (Storper \& Scott, 2009). Son escasos, sin embargo, los estudios que analizan la influencia de las representaciones regionales en la decisión de migrar dentro de un país. En este artículo se plantea que el conocer la representación social de una región aporta una dimensión relevante, pero a menudo ignorada, para entender su capacidad de atracción de nuevos migrantes. Los cambios de la realidad material de una región (salarios, empleo, infraestructuras u oportunidades de consumo) tienen un efecto limitado en los potenciales migrantes mientras no se incorporan a la representación social de dicha región, esto es, mientras no se trasladan de la dimensión socioeconómica a la sociocultural. Por ello, regiones con salarios más altos que el resto, y representadas como tales, no atraen necesariamente a los trabajadores más calificados si estos no las perciben suficientemente atractivas en otras dimensiones. Al mismo tiempo, las políticas orientadas a la dotación de infraestructuras, bienes públicos y oportunidades de consumo podrían no tener las consecuencias esperadas. Los discursos sobre las regiones poseen una profunda raíz histórica y conforman representaciones sociales que afectan la decisión de migrar, con independencia de los cambios materiales recientes.

Este artículo identifica los discursos con los que representan a la Región de Antofagasta los profesionales jóvenes de otras regiones de Chile y evalúa su influencia en los flujos de migración que recibe. Esta región, situada al norte de Chile, en el entorno del desierto de Atacama (Figura 1), se caracteriza por su fuerte especialización en la minería, que representa en torno al $60 \%$ de su producto. Durante las dos últimas décadas, ha crecido a una tasa anual del $6 \%$ y ha sido considerada por la Comisión Económica para América Latina y el Caribe (Cepal, 2009) y la Organización para la Cooperación y el Desarrollo Económicos (ocDE, 2009) como un ejemplo de región ganadora en América Latina. Sin embargo, existen síntomas de que la atracción de migrantes del resto país es menor que lo esperable.

Para estudiar su representación entre los jóvenes profesionales del país, se realizaron seis grupos de discusión con egresados universitarios en las regiones de Coquimbo, Metropolitana y de los Ríos (Figura 1), en representación de las tres zonas históricas de Chile: el Norte Chico, el Centro y el Sur, respectivamente. La información producida por estos dispositivos grupales se sometió a un Análisis Sociológico del Discurso.

Debido a su historia, geografía, especialización productiva y localización, los discursos existentes sobre la Región de Antofagasta reconocen las oportunidades de empleo y altos salarios que ofrece, pero poseen un marcado carácter negativo. A pesar de la prosperidad experimentada por esta región durante las dos últimas décadas, estos discursos podrían afectar las características de sus flujos de migración, lo cual, a su vez, influiría en sus posibilidades de desarrollo futuro.

El artículo se organiza en cinco partes. La primera plantea la conveniencia de considerar el espacio como una construcción social para entender mejor el fenómeno de la migración interna. La segunda parte presenta el caso de estudio. A continuación, se detalla la metodología empleada. En la cuarta parte se describen los discursos identificados sobre la Región de Antofagasta y, por último, en las conclusiones, se discute su potencial impacto en la migración. 


\section{Migración interna y representaciones regionales}

Los estudios sobre migración interna están dominados por una concepción del espacio como un contenedor físico. El debate actual sobre si son las oportunidades productivas o las de consumo los principales determinantes de la atracción de trabajadores calificados y del crecimiento de las ciudades (Storper \& Scott, 2009), utiliza esta concepción banal del espacio (Perroux, 1950). Desde esta perspectiva, los individuos son agentes racionales que, con cierto grado de información objetiva y actualizada, deciden sobre su desplazamiento entre dos puntos con el fin de cambiar su lugar de residencia. En este artículo se plantea que para comprender la migración interna es necesario, además, adoptar una concepción del espacio como una construcción social, donde la subjetividad es clave para entender el comportamiento de las personas y la evolución de los lugares. Un concepto útil para esta aproximación al espacio es el de representación social ${ }^{1}$, entendida como el proceso subjetivo de sustitución de un objeto material ausente (en nuestro caso, la Región de Antofagasta) por un objeto simbólico conocido ${ }^{2}$ (su representación) que ha sido socio-históricamente producido y que puede ser reproducido individual o colectivamente. La construcción social del espacio ha sido destacada por varios autores y aplicada desde distintos enfoques orientados principalmente al estudio de las ciudades (Lindón, 2007). Desde los primeros trabajos de Lynch (1960) y Lowenthal (1961), la llamada geografía de la percepción incorporó el carácter subjetivo del espacio, basándose en representaciones en la forma de mapas mentales de las ciudades, elaborados a partir de técnicas proyectivas, representados gráficamente a partir de la experiencia vivida (Vara Muñoz, 2008). La importancia de lo vivido también fue central en la forma de entender el espacio de los geógrafos humanistas, si bien aplicada con distintas técnicas. Con posterioridad, diversos enfoques, como la geografía posmoderna o el "giro cultural" de los años ochenta del siglo xx, han coincidido en la idea de que la construcción social del espacio se configura a partir del lenguaje y lo simbólico. Esta idea también se recoge en la llamada geografía de las representaciones, donde el espacio es el resultado de una fusión de lo real con lo imaginario (Bailly, 1989).

Hiernaux (2007) destaca que, en este campo de investigación, la línea de desarrollo más prometedora, y que todavía recibe poca atención, es la que vincula las representaciones con las prácticas. La mayoría de trabajos en esta dirección han estudiado las representaciones de las ciudades y su influencia en la vida cotidiana de sus habitantes. Son escasos, sin embargo, los trabajos que analizan la representación que tienen de los lugares quienes no los han vivido directamente y manejan discursos construidos históricamente, donde se mezclan la experiencia transmitida y la imaginación, una dimensión del espacio que resulta fundamental para entender las decisiones de migración interna. Aproximaciones preliminares a este problema pueden encontrarse en los trabajos de Gould y White (1974) y Fuller y Chapman (1974), quienes elaboran mapas mentales que representan gráficamente

1 El concepto de representación social fue propuesto desde la psicología social por Moscovici en la década de los sesenta, aunque tiene su origen en el concepto de representación colectiva que describió Durkheim a finales del siglo xix.

2 Compuesto por una asociación determinada de elementos (imágenes, relatos, sentimientos, etcétera). 
las preferencias reveladas por las personas sobre un conjunto de regiones alternativas de residencia. Si bien estos trabajos incorporan la subjetividad de los potenciales migrantes, tienen la limitación de mantenerse anclados en las dos dimensiones de la cartografía clásica e ignorar las representaciones sociales que se encuentran detrás de las preferencias expresadas.

Para situar el estudio de la migración interna en el marco de las representaciones sociales, resulta particularmente interesante la concepción del espacio que propone Massey $(1994,2005)$. Esta autora comparte la idea del espacio como un producto social donde se entremezclan discursos de distintos orígenes, que históricamente configuran la representación social de los lugares y que afectan las prácticas de las personas y la evolución de los lugares. Para Massey, esta concepción del espacio supone romper con la idea de un "dentro" y un "fuera" de los lugares. Esta delimitación de fronteras, señala, puede establecerse si es útil para los objetivos de la investigación, pero a su juicio no es necesaria para la conceptualización del espacio, en tanto que conjunto de historias interrelacionadas. En consecuencia, rechaza la noción según la cual los lugares tienen una única identidad, tradicionalmente vinculada a la idea de comunidad y definida solo por la introspección de sus habitantes. Por el contrario, la identidad de los lugares es reflexiva, múltiple y conflictiva. Surge también del conjunto de relaciones con el "exterior", cuyas representaciones se convierten, de esta forma, en parte de lo que es un lugar (Massey, 1994).

Esta perspectiva manifiesta la relevancia de estudiar no solo los discursos relativos a una región que poseen sus habitantes, sino también los discursos sobre dicha región de quienes no viven en ella, pues constituyen parte de su identidad y, por tanto, pueden afectar su futuro en la medida en que la región se configure como un lugar atractivo o no para vivir.

\section{Metodología}

\section{Técnica de análisis}

Como el objeto de estudio de la investigación plasmada en este artículo es la relación entre subjetividad y comportamiento y, más en concreto, entre la representación regional y la decisión de migrar, entendemos que lo más adecuado es adoptar una metodología social cualitativa y, especialmente, una técnica de conversación como el grupo de discusión. Esta técnica proporciona un acceso privilegiado a la construcción social de la realidad, pues permite reconstruir los marcos de sentido que utilizan los agentes para orientar sus prácticas sociales. Si bien es cierto que otras técnicas de conversación, como la entrevista, también logran acceder a los marcos de sentido, hacen más complejo diferenciar entre las estructuras subjetivas individuales (resultado de una trayectoria vital determinada) y las estructuras subjetivas sociales (compartidas por una población o grupo social determinado). Frente a estas técnicas de conversación, la encuesta estadística, por su estructura lógica (pregunta cerrada/respuesta cerrada), limita gravemente la producción de subjetividad por parte de los agentes, al impedirles desarrollar un relato completo (coherente y consistente) o abordar cuestiones por las que no han sido preguntados pero que 
resultan imprescindibles para la comprensión de lo que se les demanda. Hemos optado, por tanto, por la estrategia metodológica del grupo de discusión y del análisis sociológico del discurso, como han sido teorizados y puestos en práctica por la Escuela Cualitativista de Madrid ${ }^{3}$.

El grupo de discusión es un dispositivo de generación de verbalizaciones a partir de grupos experimentales. Su formato (grupos experimentales) puede resultar similar al de otros dispositivos, como el grupo focalizado o la entrevista grupal. Sin embargo, dicha similitud es solo aparente, ya que sus fundamentos teóricos y su realización práctica son totalmente diferentes. Según el Colectivo Ioé (2010), al menos cuatro características fundamentales diferencian el grupo de discusión de otras estrategias grupales de investigación social (grupo focalizado y entrevista grupal):

a. La metodología del grupo de discusión concibe el lenguaje no solo desde su función denotativa-referencial, sino que además acentúa su función connotativa, que se deriva de su propiedad afectiva (proyecta deseos), dialógica (forma un sistema), performativa (transforma la realidad) y pragmática (el contexto aporta el sentido).

b. Para esta perspectiva, el sentido se construye socialmente. En consecuencia, no es suficiente acudir a las conciencias individuales y se necesita reconstruir los marcos sociales de sentido.

c. En el grupo de discusión, el microgrupo (grupo experimental) no se reduce a la suma de los individuos que lo componen, sino que es un campo específico de la realidad social que representa a los macrogrupos sociales (clases sociales, campos sociales, generaciones, géneros, territorios).

d. Por lo anterior, existe la necesidad de que la dinámica del grupo de discusión sea abierta (no directiva) y conversacional (multidireccional), diferenciándose así del resto de dispositivos grupales de investigación social.

Las verbalizaciones producidas por los grupos de discusión (transformadas en texto mediante su transcripción literal) permitieron realizar un análisis sociológico del discurso sobre la representación de la Región de Antofagasta. El análisis, que pretende alcanzar el nivel social/hermenéutico ${ }^{4}$ que caracteriza a la Escuela Cualitativista de Madrid, se refiere a la dimensión pragmática del lenguaje, es decir, a cómo el contexto social determina el sentido. En este nivel, la unidad de análisis es el discurso 5 . El análisis sociológico (pragmático) del discurso se interesa por cómo los grupos sociales usan los discursos en sus interacciones (de conflicto, distinción, consenso, etcétera) y cuáles son las funciones y/o efectos sociales (función performativa) de estos usos. Es necesario conocer las condiciones sociohistóricas de la enunciación (producción discursiva), la posición social de los (re)productores del

Dicha escuela se constituyó en torno a la figura de Jesús Ibáńez. El desarrollo de su perspectiva metodológica se puede encontrar en Ibáñez, 1979; Ortí, 1989; Canales y Peinado, 1994; Alonso, 1998; Callejo, 2001; Gutiérrez Brito, 2008; Conde Gutiérrez del Álamo, 2009; y Colectivo Ioé, 2010.

4 Siguiendo a Alonso (1998), el primer nivel de análisis es el "informacional/cuantitativo", el segundo nivel es el "estructural/textual” y el último nivel de análisis es el "social/hermenéutico".

5 Para profundizar en las características de los discursos sociales, véase Conde Gutiérrez del Álamo (2009). 
discurso (relaciones de dominación) y el campo discursivo (conjunto de discursos en relación de interdependencia) para poder interpretar sociológicamente el sentido de lo dicho.

\section{Población de estudio}

La población de estudio son los actuales egresados universitarios chilenos, es decir, los futuros profesionales de Chile. Se considera que atraer a este colectivo es asunto estratégico para la Región de Antofagasta. Una parte importante del futuro desarrollo de la región dependerá de su capacidad para captar trabajadores de alta cualificación, tanto para cubrir las necesidades de los sectores productivos ya maduros (minería), como para posibilitar el desarrollo de nuevas actividades económicas (servicios avanzados y/o industria).

Se realizaron seis grupos de discusión limitados a tres ciudades universitarias de Chile: Coquimbo, Santiago y Valdivia (Figura 1). Los grupos de discusión, realizados entre abril y noviembre de 2010, estaban compuestos por egresados universitarios $^{6}$ de estas tres regiones, que representaban el Norte Chico, el Centro y el Sur del país, respectivamente. El diseño de dichos grupos incorporó dos dimensiones, la de género y la socioespacial, mediante los criterios de región y distancia respecto de la Región de Antofagasta (Cuadro 1). Además, se buscó la máxima heterogeneidad posible dentro de cada grupo de discusión en cuanto a la carrera y a la clase social de los participantes ${ }^{7}$, ya que ambas propiedades sociales están fuertemente relacionadas ${ }^{8}$.

\section{CUADRo 1 Diseño muestral}

\begin{tabular}{|c|l|c|l|l|l|}
\hline $\begin{array}{c}\text { GRUPOS DE } \\
\text { DISCUSIóN }\end{array}$ & GÉNERo & PARTICIPANTES & REgIÓN & DISTANCIA & \multicolumn{1}{c|}{ CARRERA Y CLASE SOCIAL } \\
\hline GD1 & Masculino & 9 & Coquimbo & Cercana & Máxima heterogeneidad posible \\
\hline GD2 & Femenino & 8 & Coquimbo & Cercana & Máxima heterogeneidad posible \\
\hline GD3 & Masculino & 11 & Metropolitana & Intermedia & Máxima heterogeneidad posible \\
\hline GD4 & Femenino & 8 & Metropolitana & Intermedia & Máxima heterogeneidad posible \\
\hline GD5 & Masculino & 12 & Los Ríos & Lejana & Máxima heterogeneidad posible \\
\hline GD6 & Femenino & 8 & Los Ríos & Lejana & Máxima heterogeneidad posible \\
\hline
\end{tabular}

FUENTE ELABORACIÓN PROPIA

6 Se consideró "egresado universitario" a estudiantes de último año de pregrado, estudiantes de posgrado y egresados con menos de dos años en el mercado laboral.

7 En este sentido, la composición social de los grupos de discusión reprodujo la composición social de la universidad chilena, esto es, las clases privilegiadas estuvieron sobrerrepresentadas, mientras que las clases populares estaban infrarrepresentadas.

8 Respecto de la dinámica, cada grupo se reunió una sola vez, utilizando como tema de convocatoria "el mercado laboral regional”, para cumplir con el requisito de aproximación indirecta al tema de estudio propio de esta técnica. 
La hipótesis del trabajo de exploración es que las propiedades sociales de género y socioespacial (región y distancia) están relacionadas con la (re)producción de discursos diferentes sobre la Región de Antofagasta. A este respecto, debe tenerse en cuenta que Coquimbo se encuentra a 910 kilómetros de Antofagasta, Santiago a 1.370 kilómetros y Valdivia a 2.210 kilómetros.

FIgura 1 | Regiones de Chile

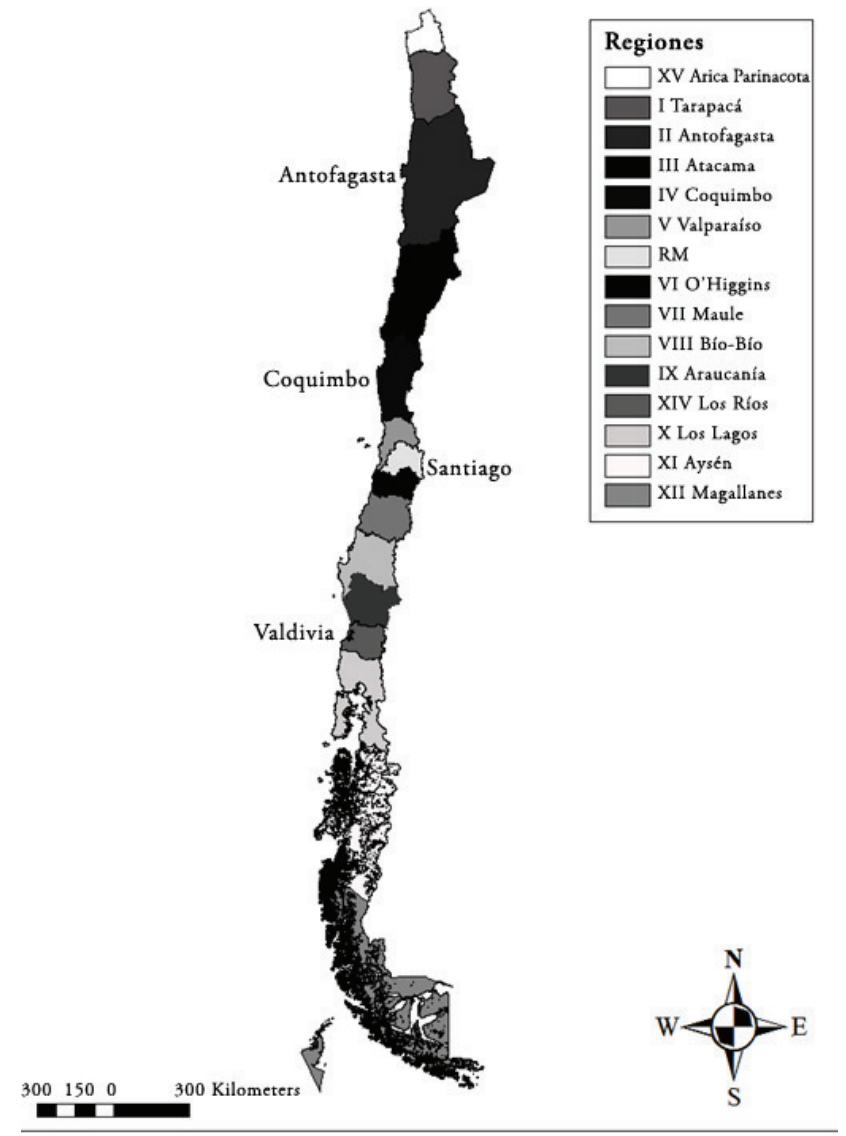

FUENTE ELABORACIÓN PROPIA

\section{Migración y conmutación hacia la Región de Antofagasta}

La Región de Antofagasta se encuentra en el norte de Chile, en el desierto de Atacama. Su población, 569.634 habitantes, representa el 3,4\% de la población de Chile (Ministerio de Planificación, Encuesta de Caracterización Socioeconómica Nacional [CASEN], 2009). Su principal actividad económica es la minería, que supone cerca del $60 \%$ de su producto y representa aproximadamente el 50\% de la producción minera nacional (Banco Central de Chile). Este porcentaje es relevante, pues Chile es el mayor productor mundial de cobre, con un 35\% del total y un $40 \%$ de las exportaciones (Comisión Chilena del Cobre [Cochilco], Cifras de 
Producción de Chile). Sin embargo, la mano de obra empleada directamente en la minería es del 16\% del total. Los flujos migratorios hacia la Región de Antofagasta tienen características singulares dentro de Chile que podrían ser, en parte, el resultado de las representaciones que se tienen de esta región. En este apartado se destacarán algunos de estos rasgos.

Según el Censo de Población y Vivienda de 2002 (Instituto Nacional de Estadísticas [INE], 2002), entre 1997 y 2002, la Región de Antofagasta ocupó el sexto lugar dentro del país en cuanto a número de trabajadores migrantes recibidos (20.594 personas), y el cuarto lugar en cuanto al porcentaje que representaban los migrantes en el total de la fuerza laboral (12\%). Si bien estas cifras son positivas, conviene matizarlas con una de las características más relevantes de los flujos de personas hacia esta región. Antofagasta destaca por ser la región de Chile con una mayor tasa neta de recepción de commuters de larga distancia, que trabajan en la región pero viven en otra (Aroca \& Atienza, 2008 y 2011). En valores absolutos, Antofagasta es la única región del país donde el número de migrantes recibidos (20.594) se aproxima al número de commuters (16.474), quienes representan casi el 10\% de su fuerza laboral. Este resultado está relacionado con la fuerte especialización minera de Antofagasta y el sistema de turnos que caracteriza esta actividad. Sin embargo, cuando se controla por la estructura sectorial ${ }^{9}$, el porcentaje de commuters que recibe la Región de Antofagasta resulta un 57\%, superior al que cabría esperar según la estructura sectorial de la conmutación nacional. Si se considera que una parte de los trabajadores que conmutan hacia Antofagasta desde otras regiones podrían convertirse en potenciales migrantes, es razonable pensar que el atractivo de la región como lugar de trabajo, vinculado principalmente a los altos salarios de la minería, no parece convertirla en un lugar atractivo para vivir.

Otro rasgo característico de los flujos de migración y conmutación hacia Antofagasta es su escaso atractivo para las mujeres. Es la región de Chile que recibe un menor porcentaje de mujeres migrantes, un 26,4\% del total regional, solo por detrás de la Región de Magallanes. Antofagasta está especializada en la recepción de varones, tanto migrantes como commuters, mientras que recibe cerca de un $20 \%$ menos mujeres migrantes y tres veces menos mujeres commuters que el promedio del país (Cuadro 2). Es destacable que estos resultados se mantienen, aunque se suavizan ligeramente, cuando se controla por la estructura sectorial de la región. Antofagasta recibe un $11 \%$ menos de mujeres migrantes y menos de la mitad de mujeres commuters que las que cabría esperar teniendo en cuenta su estructura productiva (Cuadro 2). reales y esperados de los commuters para cada una de las distintas actividades de la región, dividida entre el total del empleo que los commuters representan en la región. El valor esperado corresponde a la distribución de commuters por sector que se observa en el conjunto del país. 
CUADRo 2 Cocientes de localización de migrantes y commuters según sexo*

\begin{tabular}{|c|c|c|c|c|}
\cline { 2 - 5 } \multicolumn{1}{c|}{} & \multicolumn{2}{|c|}{ COCIENTE DE LOCALIZACIÓN } & \multicolumn{2}{c|}{$\begin{array}{c}\text { COCIENTE DE LOCALIZACIÓN } \\
\text { CONTROLANDO POR ESTRUCTURA } \\
\text { SECTORIAL }\end{array}$} \\
\cline { 2 - 5 } & Migración & Conmutación & Migración & Conmutación \\
\hline Hombres & 1,09 & 1,12 & 1,04 & 1,1 \\
\hline Mujeres & 0,81 & 0,31 & 0,89 & 0,47 \\
\hline
\end{tabular}

FUENTE ELABORACIÓN PROPIA A PARTIR DEL CENSO 2002

*Valores por encima de 1 implican sobrerrepresentación y por debajo subrepresentación.

Tanto los flujos de migración como los de conmutación hacia la Región de Antofagasta se caracterizan por estar especializados en la recepción de técnicos y, especialmente, de operarios. La llegada de trabajadores no calificados es significativamente inferior al promedio nacional, mientras que los profesionales se encuentran próximos al promedio. Al analizar el nivel educativo de los migrantes y commuters, se obtienen resultados coherentes con los anteriores, ya que la región se especializa en la recepción de trabajadores con educación media, que representan el $47 \%$ de los migrantes y el $56,5 \%$ de los commuters, mientras que se encuentra próxima al promedio nacional en el caso de la educación técnica y universitaria.

Por último, cabe destacar el significativo efecto de la distancia en el decaimiento de los flujos de migración hacia la Región de Antofagasta. Entre 1997 y 2002, el $45,5 \%$ de los migrantes provino del Norte (regiones I, III y IV); el 26,8\% de la Región Metropolitana; el 22,6\% de la Zona Central (regiones v, vi, viI y viII); y apenas un 5,1\% de la Zona Sur (regiones IX, X, XI y XII). La opción de conmutar hacia la Región de Antofagasta también se reduce de forma relevante con la distancia y tiene dos focos principales, el Norte Grande con un 46,8\% de los commuters y la Región Metropolitana, con un 21,4\%.

\section{Representación socioespacial de la Región de Antofagasta ${ }^{10}$}

Un primer rasgo destacable de los discursos sobre la migración regional es la representación de los lugares principalmente a partir de un esquema de valoración e interpretación funcional que responde a la pregunta ¿qué nos puede aportar esa región? En este sentido, el eje semántico fundamental que organiza las distintas representaciones es el de Calidad de vida vs. Oportunidades laborales. Desde esta perspectiva, la Región de Antofagasta se construye siempre en comparación con el resto de regiones del país. Junto a esto sobresalen las marcadas diferencias en la extensión y complejidad con que representan a la Región de Antofagasta las tres regiones donde se realizó el trabajo. Como cabía esperar, la complejidad de las representaciones está determinada por la importancia social de la región, que depende fundamentalmente de dos factores: la cercanía socioespacial con la región de residencia y la relevancia de esta región en la construcción sociohistórica de Chile. 
La cercanía socioespacial se refiere a la distancia geográfica con la región de residencia, a los nexos sociales con Antofagasta (personas conocidas que viven en ella o la conocen) y a la posibilidad subjetiva de migrar a esta región. La representación de la Región de Antofagasta es más extensa y compleja en los grupos de discusión de la Región de Coquimbo (máxima cercanía espacial, abundantes nexos sociales y mayor probabilidad de migrar), más simple en la Región Metropolitana (más lejana y con pocas probabilidades de migrar) y casi inexistente y muy metafórica en la Región de Los Ríos (donde "el norte" es "Santiago").

La construcción sociohistórica del país ha dado pesos muy diferentes a las regiones en su aportación al discurso nacional de Chile, a lo que se considera la chilenidad. El Chile auténtico está compuesto por el centro y centro-sur del país, mientras que el extremo sur (Región de Magallanes) y el desierto de Atacama (Regiones de Atacama, Antofagasta, Tarapacá, y Arica y Parinacota) son territorios ocupados posteriormente por el Chile auténtico y que necesitan ser chilenizados (colonizados). Como consecuencia de lo anterior, las regiones del centro y centrosur tienen representaciones más densas y cristalizadas que las regiones periféricas, pues la socialización nacional (la chilenización) se focaliza en el conocimiento y reconocimiento del Chile auténtico. Este factor explica, entre otras cosas, que los grupos de discusión de Coquimbo reproduzcan una representación del "sur" mucho más desarrollada que la representación que los grupos de discusión de Valdivia realizan del "norte" (casi ausente).

Dadas las diferencias encontradas entre las distintas regiones sobre la representación de Antofagasta, este apartado recogerá por separado los discursos de cada una de ellas, para posteriormente realizar una síntesis de los discursos identificados.

\section{Representación socioespacial desde la Región de Coquimbo}

Desde Coquimbo, Chile aparece dividido en cuatro grandes regiones simbólicas: "Coquimbo-Serena", "Santiago", el "sur” y el "norte". La región simbólica de "Coquimbo-Serena" es representada exclusivamente por el discurso de región dormitorio. Este concepto condensa una gran cantidad de dimensiones donde domina su función social: "es la región ideal para vivir, no para trabajar". Se argumenta que, por el clima, la calidad medioambiental, el nivel de seguridad y su belleza, esta región sería el mejor sitio para vivir y tener una familia. El gran problema de esta región es que no tiene suficiente trabajo y la gente se ve obligada a migrar. En el caso de Santiago, el eje central que atraviesa todas las dimensiones que componen su representación es la necesidad de ser joven y soltero o soltera para aprovechar y soportar la ciudad. "Santiago" es representado como el mejor lugar del país para desarrollarse profesionalmente, debido a una gran oferta educativa y laboral. También destaca por su gran oferta de ocio y cultura, la mayor del país. Sin embargo, tendría una baja calidad de vida debido a las grandes distancias de transporte, la contaminación y la inseguridad. Por su parte, la representación de la región simbólica del "sur" es la menos desarrollada por los grupos de discusión de Coquimbo y la más metafórica (condensa muchos significados), debido a la gran distancia socioespacial que las separa. Se centra en tres dimensiones: la estética, la de sociabilidad y la climática. El "sur" sería el lugar más bello y 
turístico de Chile, una especie de paraíso natural y social donde todo es más bello, natural, tranquilo y saludable. El problema del "sur" es su mala climatología, por lo que sería un lugar más atractivo para veranear que para residir.

Con estos marcos geográficos de referencia, la representación de la región simbólica del "norte", que incluye la Región de Antofagasta, desde la Región de Coquimbo es bastante compleja y desarrollada debido a la cercanía socioespacial. Dicha representación está determinada tanto por la función social que cumple, como por sus carencias. "El norte es solo plata", lo que se relaciona con los altos ingresos de la gran minería, pero, al mismo tiempo, se describe como un lugar sin naturaleza, feo y aburrido para la mayoría de la gente, aunque dicen conocer personas que afirman que les gusta (gusto excéntrico). Puede resultar interesante ver el desierto una vez en la vida, pero vivir en él sería horrible. Las mineras tienen que pagar sueldos más altos que en el resto del país para compensar la pérdida de calidad de vida por habitar en mitad del desierto. Se subraya que el "norte" es solamente para trabajar: "el dinero que ganan los mineros se lo gastan en otras regiones".

Para los egresados de la Región de Coquimbo, las principales "ciudades mineras" del "norte" son Calama, Tocopilla, Copiapó y Antofagasta. Estas ciudades son representadas de forma muy negativa, aunque con énfasis diferentes según el género. Tanto hombres como mujeres coinciden en caracterizarlas como ciudades extremadamente feas, debido a la ausencia de vegetación e infraestructura urbana y a que las casas "están sin acabar". También son vistas como ciudades muy caras, lo que se atribuye a la imposibilidad de producir nada en el desierto (esterilidad productiva). Una de las características negativas más importantes que distinguiría a las "ciudades mineras" es su elevada delincuencia, producto, por un lado, del tráfico de drogas concentrado en las fronteras con Perú y Bolivia; y por otro, de los altos ingresos que ofrece la minería. El grupo de discusión masculino enfatiza el problema de la falta de servicios "mínimos" de entretención, como cines o centros comerciales, y considera peligrosa la contaminación producida por la explotación minera, mientras que el grupo de discusión femenino se centra más en el aspecto estético, asegurando que son ciudades caóticas y muy sucias.

La condición de "ciudades" es muy cuestionada, pues su existencia está relacionada con el auge minero. Las "ciudades mineras" durarán lo que duren los yacimientos de cobre. Este carácter de provisionalidad explica, para los egresados de la Región de Coquimbo, que las personas que se desplazan hasta allí a trabajar no piensen en establecerse definitivamente. El carácter perecedero o finito de la explotación minera es atribuido por asociación a las "ciudades mineras", que son representadas como ciudades-campamento, cuya forma típica de organizar el trabajo es el sistema de turnos. Dicho sistema no permite a los trabajadores hacer vida familiar, porque los obliga a vivir en campamentos alejados de las ciudades. Como la convivencia familiar no es posible, las familias prefieren vivir en la Región de Coquimbo, que, además de tener mucha más calidad de vida, es más barata que las "ciudades mineras".

Las egresadas de Coquimbo representan las "ciudades mineras" como ciudades masculinizadas ("hechas para hombres") y proletarizadas, pobladas por una masa de hombres que llegaron sin nada, procedentes de otras regiones y países, atraídos por la idea de "hacer plata" en la minería. Una versión chilena del Far West producida 
por la fiebre del cobre. Los servicios de estas ciudades están dirigidos a la atención de este tipo de hombres mediante la oferta de comercio sexual, consumo de alcohol y otras drogas en diversos espacios de sociabilidad eminentemente masculina. La gran demanda de mujeres para estos servicios de entretención masculina habría producido una fuerte inmigración femenina proveniente de otros países latinoamericanos, estableciéndose una relación directa entre inmigración femenina y prostitución.

Para ellas, el perfil típico de las personas que se van a trabajar a las ciudades mineras (aparte de los profesionales de la minería), es el de hombres de "clase baja" sin estudios universitarios que buscan tener una capacidad adquisitiva ("estándar de vida") superior a la que les corresponde por su nivel educativo y ocupacional. Hay una valoración negativa de este tipo de emigrante, construida a partir de un esquema clasista, racionalizada mediante la ideología liberal de la meritocracia escolar. El arquetipo de este tipo de emigrante es el de los "rotos con plata", personas "sin clase" que han conseguido injusta y coyunturalmente altos ingresos, que gastan rápida e irracionalmente en drogas, prostitución y ejercicios de ostentación. Por su parte, los habitantes del "Norte Grande" son representados -con una connotación negativacomo insociables y racialmente altiplánicos. Estas características los diferenciarían del resto de chilenos, pero sin que ello signifique que se ponga en duda su chilenidad.

En resumen, podemos decir que las "ciudades mineras" son representadas como espacios antiestéticos, antifamiliares, caros y muy inseguros, además de aburridos para los egresados e inmorales para las egresadas (Cuadro 3).

\section{CUADRO 3 | Las ciudades mineras}

\begin{tabular}{|c|c|c|}
\hline $\begin{array}{l}\text { DIFERENCIAS DE } \\
\text { GÉNERO }\end{array}$ & CARACTERÍSTICAS & PROBLEMAS \\
\hline \multirow{4}{*}{ Consenso } & Ciudades feas & Estético \\
\hline & Ciudades caras (esterilidad productiva) & Precios \\
\hline & Ciudades sin posibilidad de vida familiar & Familiar \\
\hline & Alta delincuencia (drogas) & \multirow{3}{*}{ Seguridad } \\
\hline \multirow{4}{*}{ Énfasis masculino } & Contaminación (residuos de las mineras) & \\
\hline & Ciudades provisionales (ciudad-campamento) & \\
\hline & Ciudades sin entretención & Ocio \\
\hline & Ciudades explotadas (centralismo chileno) & Inversión pública \\
\hline \multirow{5}{*}{ Énfasis femenino } & Ciudades sucias & Estético \\
\hline & Ciudades semiconstruidas (pseudo-ciudades) & Infraestructuras \\
\hline & Ciudades masculinizadas (prostitución) & \multirow{3}{*}{ Moral } \\
\hline & Ciudades consumistas (ostentación) & \\
\hline & Ciudades proletarizadas ("rotos con plata") & \\
\hline
\end{tabular}

FUENTE ELABORACIÓN PROPIA 
La gran minería es un elemento recurrente en los discursos sobre las oportunidades de empleo que ofrece la Región de Antofagasta, respecto del cual emergen dos posiciones discursivas, determinadas también por el género. La masculina está caracterizada por una fuerte ambivalencia entre los valores materialistas, por un lado, y los valores familiares (tradicionales), ecológicos y políticos (posmaterialistas), por otro. En contraste, la posición femenina destaca los roles de género tradicionales (patriarcales) del sector minero y las consecuencias de la organización del trabajo (sistema de turnos) en la vida familiar.

Los varones reconocen que les resultan muy atractivas las grandes empresas del sector minero. Los altos sueldos y el sistema de trabajo por turnos son sus principales atractivos. Con altos ingresos "puedes viajar a donde quieras" y el sistema de turnos "deja muchos días libres para hacer lo que quieras". Además, aseguran que el sector minero "es el único que cumple totalmente con la legislación laboral". Sin embargo, dicen ser muy conscientes de todo el "daño medioambiental" que produce. Una estrategia que emerge para lidiar con esta ambivalencia es aceptar a corto plazo trabajar en el sector y, a largo plazo, tras satisfacer las necesidades materiales, encontrar una ocupación que les permita "luchar contra las mineras". Otro costo del que dicen ser conscientes es la pérdida de la vida familiar. El trabajo por turnos es muy atractivo mientras están solteros, pero cuando tengan familia se perderán "la mitad de la vida de sus hijos". Es representado, por tanto, como un sector antifamiliar. Por último, los egresados acusan a "las mineras" de "llevarse toda la plata que ganan a Santiago o fuera del país”. Esta crítica político-económica señala, por un lado, que la minería sigue la lógica estatal del centralismo, que concentra recursos en "Santiago" y empobrece las "regiones"; y, por otro, la de la explotación internacional, que enriquece a los países del centro empobreciendo a los de la periferia.

Los discursos femeninos caracterizan la minería como un universo masculino y antifemenino. Dicen ser conscientes de que en el sector minero los ingresos son más altos, pero les resulta un mundo repelente, solo apto para los "hombres". Además, a diferencia del grupo de discusión masculino, piensan que las condiciones laborales en la minería son duras y desagradables, especialmente la organización del trabajo por turnos, que las aleja, entre otras cosas, de su familia de origen. Los esquemas de apreciación de la minería, en el caso de las egresadas, están determinados por la diferencia de género y por la funcionalidad del sector para la vida familiar, percibiéndose totalmente disfuncional.

Las representaciones socioespaciales que emergen en la Región de Coquimbo pueden representarse en un mapa topológico a partir de tres ejes semánticos (Figura 2). El primero (eje vertical) ordena el territorio a partir de su relevancia política (centro/frontera) y de su nivel de desarrollo (civilización/barbarie), donde las ciudades son menos importantes y desarrolladas cuanto más alejadas están de Santiago; el segundo eje (horizontal) ordena el territorio a partir de su entorno medioambiental (mar/desierto) y valoración estética (belleza/fealdad), donde las ciudades son menos bellas cuanto más adentradas estén en el desierto; y por último, un tercer eje (diagonal) que ordena el territorio a partir de su función social (calidad de vida/plata), donde hay ciudades que son ideales para vivir (región dormitorio) y otras ideales para lucrar (norte minero). 
FIgURA 2 | Representación socioespacial de las regiones desde la Región de Coquimbo

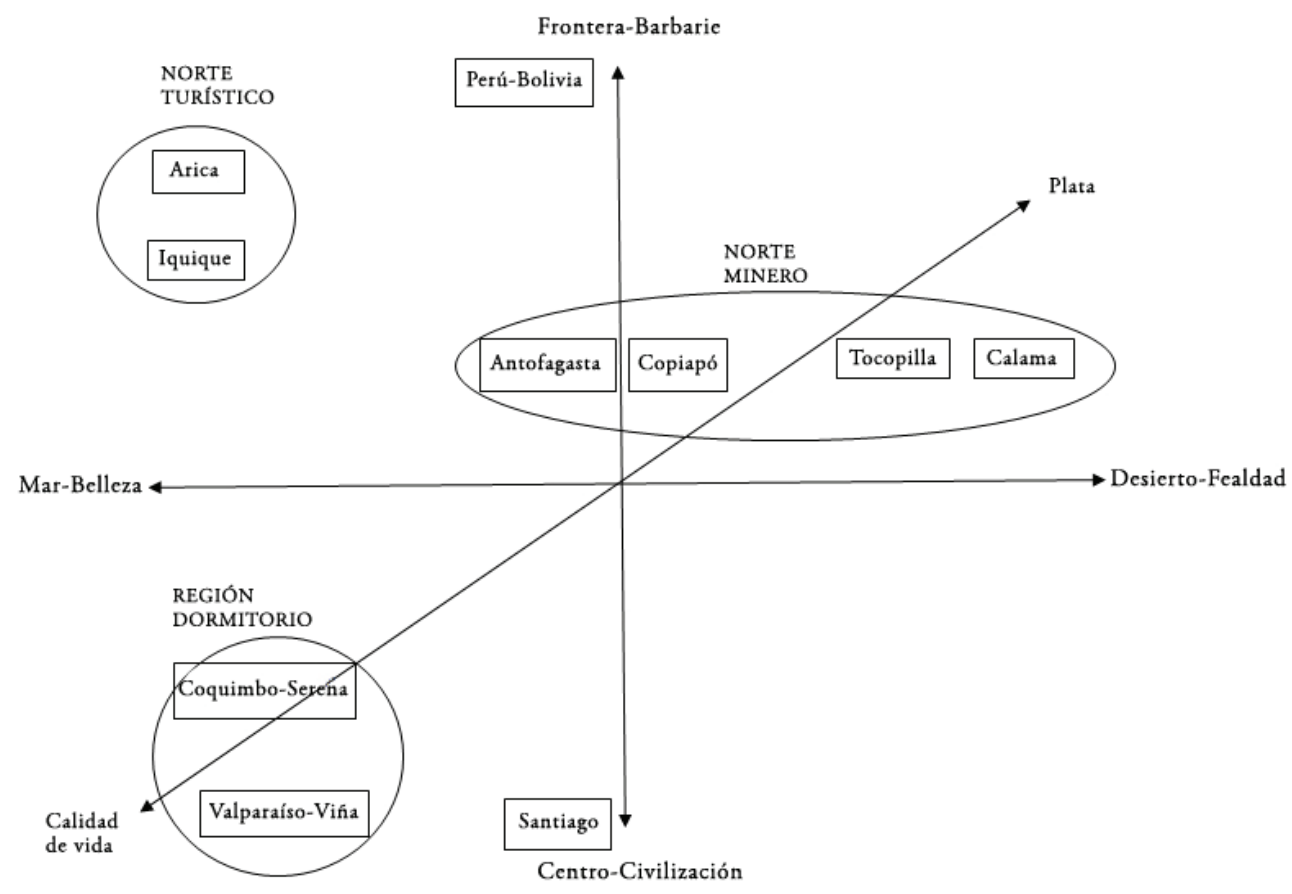

FUENTE ELABORACIÓN PROPIA

\section{Representación socioespacial desde la Región Metropolitana}

La representación de la Región de Antofagasta se simplifica en la Región Metropolitana en comparación con Coquimbo, prevaleciendo los discursos más negativos. Desde esta región se describen tres grandes regiones simbólicas: "Santiago", el "norte" y el "sur". "Santiago" se representa a partir del discurso centralista (Rodrigo, 2011), esto es, dispondría de una superioridad multidimensional frente al resto de Chile. Precisamente esta superioridad haría poco atractiva la migración regional para sus habitantes. El "sur" de Chile es representado como un paraíso natural, pero con diferentes niveles de desarrollo y de atractivo laboral. Se distingue entre:

a. El sur extremo, compuesto por Puerto Montt, Puerto Varas, Punta Arenas y Coyhaique, con mejores ingresos que Santiago producto del esfuerzo público por mantener allí la soberanía, pero con un clima extremo que impide la sociabilidad, existiendo altas tasas de alcoholismo y suicidio.

b. El sur pobre: compuesto por Valdivia y Chiloé. Considerados lugares bellos pero con pocos recursos y alto desempleo.

c. El sur próximo: compuesto por Concepción y Temuco. Es la mejor zona para migrar porque, además de la cercanía a Santiago, tendría un buen desarrollo económico y de servicios, con sueldos y oportunidades laborares superiores a Santiago. 
d. El "norte" es representado de forma muy esquemática y sin las connotaciones positivas del "sur". En los discursos, se divide en dos zonas a partir de su desarrollo económico:

i) El norte pobre: compuesto por Arica e Iquique, dos ciudades turísticas pero muy pobres y con mucho desempleo;

ii) El norte minero: compuesto por Antofagasta, Copiapó y Calama. En estas ciudades, podrían tener ingresos mayores que en Santiago debido al sector minero. El problema es que se reproduce la representación de las ciudades mineras para describir el espacio, y la de los rotos con plata para describir a sus habitantes.

En general, los nortinos son percibidos como insociables, racial y culturalmente altiplánicos y menos chilenos que el resto, por estar peruanizados.

Las representaciones socioespaciales producidas en la Región Metropolitana quedarían organizadas en un mapa topológico con solo dos ejes semánticos (Figura 3). Un eje de "ingresos" (vertical) y otro de "calidad de vida" (horizontal). Existe un territorio con altos ingresos y baja calidad de vida (zonas extremas), donde se encuentra la Región de Antofagasta; un segundo territorio con buenos ingresos y buena calidad de vida (zonas residenciales); y un tercer territorio empobrecido, pero con alta calidad de vida (zonas turísticas).

FIGURA 3 | Representación socioespacial de las regiones desde la Región Metropolitana

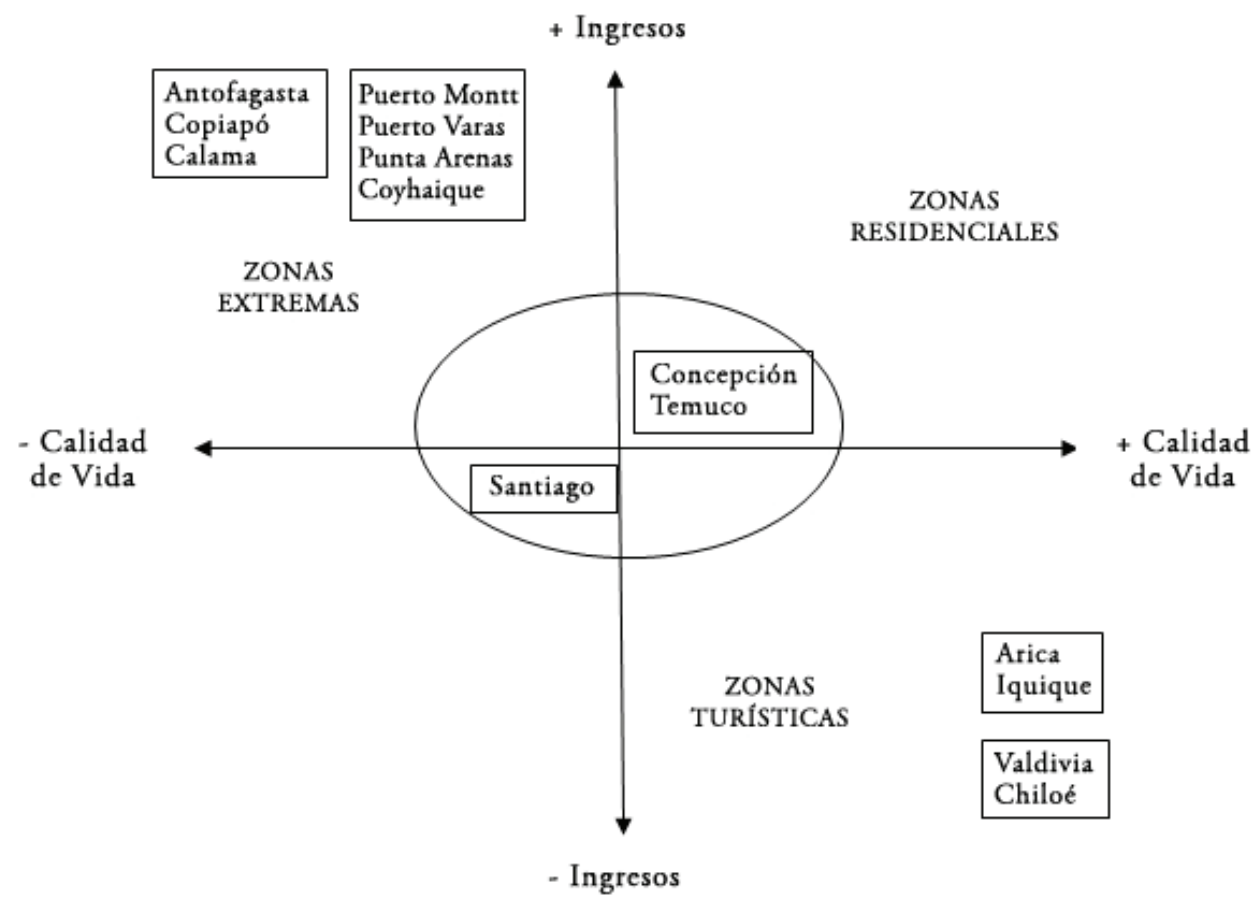

FUENTE ELABORACIÓN PROPIA 


\section{Representación socioespacial de las regiones desde la Región de Los Ríos}

En esta región, Chile queda dividido en cuatro grandes regiones simbólicas: "Valdivia", el "sur", el "norte" y las "zonas extremas". La Región de Antofagasta prácticamente desaparece, confundida con otros espacios bajo la denominación de "zonas extremas". "Valdivia" es el mejor lugar para vivir y desarrollar un proyecto familiar. Es la primera opción residencial por su calidad medioambiental, belleza, sociabilidad de sus habitantes y tamaño accesible. Sin embargo, su mercado laboral "saturado" hace muy difícil, para la mayoría de los egresados, desarrollar allí su carrera profesional, obligando a una gran parte a migrar. Dada esta necesidad, emergen tres destinos posibles (aunque solo dos probables): el "sur", donde va la mayoría de la gente; el "norte", donde iría un grupo menor; y las "zonas extremas", un destino muy improbable y limitado a los no profesionales.

a. El sur: compuesto principalmente por Osorno y Puerto Montt, es el destino principal de los egresados de la región. Se consideran ciudades con más empleo y servicios que Valdivia. Además, son cercanos (Osorno está a solo $110 \mathrm{~km}$ y Puerto Montt a $210 \mathrm{~km}$ ) y tienen una calidad de vida similar y muy superior a Santiago y a las "zonas extremas".

b. El norte: hace referencia a Temuco, Concepción y Santiago, siendo destinos secundarios adonde se dirige un grupo menor de egresados. Santiago es especialmente atractivo, por concentrar a todas las grandes empresas, tener una demanda continua de profesionales y sueldos más altos que Valdivia. Sin embargo, su problema es la lejanía de Valdivia $(840 \mathrm{~km})$ y una mala calidad de vida en comparación con Temuco y Concepción, considerados mejores destinos.

c. Las zonas extremas: recogen un conjunto de lugares representados como muy lejanos, aislados, con un clima hostil y pocos servicios. En esta categoría está Coyhaique, Cochrane, Toltén y Puerto Natales, hacia el extremo sur; y Antofagasta, Tocopilla, Copiapó y Calama, hacia el extremo norte. En las "zonas extremas" se pagarían sueldos muy altos y gozarían de ciertas exenciones fiscales como una estrategia estatal para atraer población ("allí vas a colonizar, a hacer patria”). El extremo norte se percibe atractivo para los trabajadores no profesionales, porque la minería genera puestos de trabajo no calificados con sueldos altos. De cualquier forma, es muy poca la gente que se decide por estos destinos, porque tendrían la peor calidad de vida de todo Chile y están muy alejadas.

d. Los destinos se organizan a partir de dos ejes (Figura 4): un eje funcional (calidad de vida ${ }^{11}$ /estándar de vida) y el eje de desarrollo (civilización/colonización). Obtenemos así un territorio donde se puede alcanzar un alto estándar de vida, pero que aún está por desarrollar (zonas extremas): aquí está ubicada la Región de Antofagasta; existe un segundo territorio que está muy desarrollado y que también permite obtener un alto estándar de vida (Santiago y Concepción); por

11 Por "calidad de vida" se refiere al conjunto de dimensiones que consideran determinantes de su bienestar: el nivel de estrés, la distancia entre el trabajo y la vivienda, el tiempo disponible para la familia y ocio, el clima, la calidad medioambiental, la cercanía espacial con la familia y la seguridad ciudadana. Se opone al concepto de "estándar de vida", referido a la capacidad adquisitiva o al nivel de consumo. 
último, hay otro territorio suficientemente desarrollado que, además, permite obtener una alta calidad de vida (Valdivia y Puerto Montt).

FIGURA 4 | Representación socioespacial de las regiones desde la Región de Los Ríos

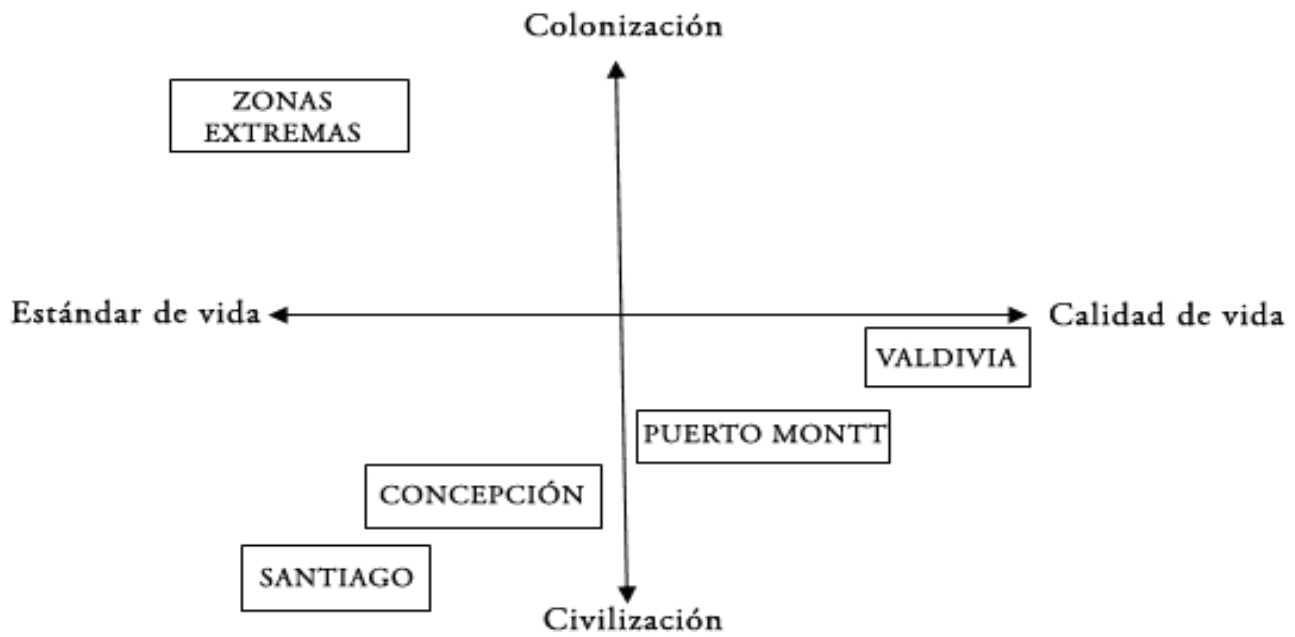

FUENTE ELABORACIÓN PROPIA

Discursos sobre la Región de Antofagasta

Como se ha podido comprobar, la Región de Antofagasta no cuenta con una representación equivalente que la designe a lo largo del país, sino que queda integrada en regiones simbólicas que se refieren siempre a espacios más amplios, como el "norte", el "norte minero", la "zona minera" o las "zonas extremas". La región simbólica del "norte" incluye las regiones administrativas de Arica y Parinacota, Tarapacá, Antofagasta y Atacama, y es utilizada por los egresados de la Región Metropolitana. La región simbólica "zonas extremas" hace referencia a las mismas regiones administrativas que la anterior, además de a las regiones de Aysén y Magallanes, siendo utilizada por los egresados de la Región de los Ríos. Por último, la región simbólica del "norte minero" o "zona minera" es la más acotada. Se refiere exclusivamente a las regiones de Antofagasta y Atacama, siendo utilizada por los grupos de discusión de las regiones de Coquimbo y Metropolitana.

Los distintos discursos que aparecieron sobre la Región de Antofagasta se pueden sintetizar en tres grandes posiciones discursivas: ambivalente, de rechazo y de ausencia (Cuadro 4). Estas posiciones están determinadas por la distancia geográfica y social con la Región de Antofagasta, siendo la posición ambivalente la más cercana (Región de Coquimbo), la posición de rechazo la intermedia (Región Metropolitana) y la posición de ausencia la más lejana (Región de Los Ríos).

\section{La posición ambivalente: Región de Coquimbo}

En esta posición coexiste una representación medioambiental, urbana y social muy negativa de la Región de Antofagasta, con una representación muy positiva de las condiciones laborales de las grandes mineras que se encuentran en la región. Estas 
representaciones generan rechazo en cuanto a residir en la región y atracción por sus oportunidades laborales. Por ello, la estrategia socioespacial que se utilice intentará obtener el máximo provecho laboral, pero evitando en lo posible la residencia.

Los discursos que componen la posición ambivalente son los más complejos y desarrollados, debido a su mayor cercanía socioespacial con la Región de Antofagasta. Representan una región medioambientalmente hostil (el norte es desierto), con ciudades antiestéticas, caras y peligrosas, y, además, con fuertes carencias en servicios e infraestructura urbana (ciudades mineras). Los habitantes de estas ciudades mineras son representados como inmigrantes nacionales y extranjeros no cualificados que buscan altos ingresos en la minería, que después gastan inmoral e irracionalmente (los "rotos con plata"). Aunque se describe a los habitantes como racialmente diferentes (altiplánicos), étnicamente son considerados chilenos.

Ante el rechazo a residir en la Región de Antofagasta, la única posibilidad de trabajar en ella, para los egresados masculinos que estén relacionados con la minería, es aprovechar el sistema de turnos, generalizado en el sector, para conmutar. La imposibilidad de trabajar por turnos hace que una oferta laboral deje de ser atractiva.

\section{La posición de rechazo: Región Metropolitana}

Frente a la ambivalencia, la posición de rechazo se caracteriza por una representación netamente negativa de la Región de Antofagasta, donde los altos ingresos que ofrece el sector minero solo confirman la enorme pérdida de calidad de vida que significaría la migración. En este sentido, el traslado hacia esta región se concibe como la última opción, una estrategia radical y desesperada que debería durar el menor tiempo posible.

La Región de Antofagasta vuelve a ser representada negativamente en la Región Metropolitana tanto en lo medioambiental (el norte es desierto), como en lo urbano (las ciudades mineras) y en lo social (los "rotos con plata"), desapareciendo la compleja representación de las condiciones laborales del sector minero y, por tanto, desapareciendo el conflicto y sus estrategias de solución (conmutación).

Los habitantes de la región son representados racial y étnicamente como altiplánicos, más próximos a peruanos y bolivianos que a chilenos.

\section{Posición de ausencia: Región de Los Ríos}

A diferencia de las anteriores, en esta posición la Región de Antofagasta no está entre los destinos laborales posibles (pensables) y prácticamente no existe como región simbólica diferenciada, encontrándose diluida y confundida con otras regiones de los extremos sur y norte de Chile bajo la nominación de "zonas extremas". La representación de la Región de Antofagasta aparece aquí difusa y metafórica, relacionada con la idea de naturaleza extrema y hostil, que comparten tanto el desierto de Atacama como la Antártica chilena.

Los habitantes de la Región de Antofagasta se representan como étnicamente más parecidos a los habitantes de los países fronterizos (Perú y Bolivia) que a los chilenos. Lo anterior se explica porque los habitantes de las "zonas extremas" (en el norte y en el sur) se encuentran muy alejados del resto de chilenos y muy próximos a los países vecinos. 
Desde esta posición desaparece la representación de las ciudades mineras. Esta ausencia denota el desconocimiento, la carencia de importancia y, sobre todo, la enorme distancia socioespacial que tienen los egresados de la Región de Los Ríos con la Región de Antofagasta.

CUADRo 4 Estructura del campo discursivo sobre la Región de Antofagasta

\begin{tabular}{|c|c|c|c|}
\hline \multirow{2}{*}{$\begin{array}{c}\text { DISTANCIA ESPACIAL } \\
\text { POSICIÓN }\end{array}$} & \multicolumn{2}{|l|}{ MÁS CERCANO } & MÁS LEJANO \\
\hline & AMBIVALENTE & RECHAZO & AUSENCIA \\
\hline Región de Antofagasta & $\begin{array}{l}\text { Coexistencia de una } \\
\text { representación urbana, } \\
\text { medioambiental y social } \\
\text { muy negativa con las me- } \\
\text { jores condiciones labores } \\
\text { del país (sector minero). }\end{array}$ & $\begin{array}{l}\text { Representación urbana, } \\
\text { medioambiental y social } \\
\text { muy negativa que se } \\
\text { intenta compensar con } \\
\text { altos ingresos. }\end{array}$ & $\begin{array}{l}\text { No forma parte de la } \\
\text { representación espacial } \\
\text { habitual de las regiones. } \\
\text { Se representa confundida } \\
\text { junto con otras regiones } \\
\text { como "zonas extremas" } \\
\text { (muy lejanas y sin } \\
\text { "civilización") }\end{array}$ \\
\hline $\begin{array}{l}\text { Habitantes de la } \\
\text { Región de Antofagasta }\end{array}$ & $\begin{array}{l}\text { Representados como más } \\
\text { insociables que el resto } \\
\text { de chilenos y racialmente } \\
\text { altiplánicos. }\end{array}$ & $\begin{array}{l}\text { Representados como más } \\
\text { insociables que el resto } \\
\text { de chilenos. Cultural y } \\
\text { racialmente altiplánicos. }\end{array}$ & $\begin{array}{l}\text { Representados como más } \\
\text { insociables que el resto } \\
\text { de chilenos. Comparten } \\
\text { la cultura de los países } \\
\text { vecinos. }\end{array}$ \\
\hline Ciudad de Antofagasta & $\begin{array}{l}\text { Representada como la } \\
\text { mejor ciudad minera. }\end{array}$ & $\begin{array}{l}\text { Representada como la } \\
\text { mejor ciudad minera. }\end{array}$ & No representada. \\
\hline $\begin{array}{l}\text { Habitantes de las } \\
\text { ciudades mineras }\end{array}$ & $\begin{array}{l}\text { Representados como pro- } \\
\text { letarizados, incultos, muy } \\
\text { vulgares y consumistas } \\
\text { irracionales. }\end{array}$ & $\begin{array}{l}\text { Representados como pro- } \\
\text { letarizados, incultos, muy } \\
\text { vulgares y consumistas } \\
\text { irracionales. }\end{array}$ & No representados. \\
\hline Chilenidad & Son chilenos & $\begin{array}{l}\text { Peruanizados, menos } \\
\text { chilenos que el resto. }\end{array}$ & $\begin{array}{l}\text { Identidad compartida } \\
\text { con los países vecinos, } \\
\text { son poco chilenos. }\end{array}$ \\
\hline Migración & $\begin{array}{l}\text { Posibilidad de conmuta- } \\
\text { ción para los egresados } \\
\text { masculinos relacionados } \\
\text { con el sector minero. }\end{array}$ & Improbable & Impensable \\
\hline $\begin{array}{l}\text { Características de la } \\
\text { representación }\end{array}$ & Muy compleja y descriptiva & Simple y descriptiva & Difuminada y metafórica. \\
\hline Origen del discurso & REGIÓN DE COQUIMBO & REGIÓN METROPOLITANA & REGIÓN DE LOS RÍOS \\
\hline
\end{tabular}

FUENTE ELABORACIÓN PROPIA

\section{Conclusiones}

Los resultados obtenidos confirman que las propiedades socioespaciales (región y distancia) y de género producen distintos discursos sobre la Región de Antofagasta, no resultando para este caso significativa la carrera o la clase social de los componentes de los grupos. La Región de Antofagasta, en ninguno de los casos, es identificada por sus fronteras administrativas, sino que es representada dentro de áreas simbólicas más amplias que la contienen. Asimismo, como se esperaba, la representación de Antofagasta resulta múltiple y conflictiva, definida en relación con el resto país, y su visión parece reproducir el patrón centro-periferia con el que se configuró históricamente el Estado chileno. 
Todos los discursos sobre la Región de Antofagasta mantienen una denotación negativa más o menos matizada. Desde la perspectiva socioespacial se pasa de una posición discursiva ambivalente en Coquimbo, positiva en lo laboral y negativa en lo residencial, a una posición marcadamente negativa en Santiago, para pasar a una representación difusa y metafórica en el sur. En todos los casos, la Región de Antofagasta se destaca como un lugar precario y poco atractivo para vivir y solo interesante por sus oportunidades laborales con ingresos altos.

Los resultados obtenidos dejan abiertas algunas líneas de investigación relacionadas con la génesis histórica de los discursos identificados y con los factores que, según los discursos, podrían estar influyendo en las decisiones individuales de migrar. Los discursos identificados contribuyen, sin embargo, a entender las altas tasas de commuters que recibe la Región de Antofagasta desde otras regiones. De hecho, en las tres regiones analizadas el porcentaje de commuters que se dirigen a Antofagasta es significativamente superior al de migrantes, destacando especialmente la Región de Coquimbo, que es la que envía un mayor porcentaje de commuters a Antofagasta.

Otro de los ejes que articula la formación de discursos sobre la Región es el género: mujeres y hombres poseen valoraciones diferentes de la región. Entre las primeras predomina una valoración netamente negativa, donde destaca la falta de estética y atractivo para vivir y desarrollar una vida familiar satisfactoria en las ciudades mineras, y la conformación de una sociedad masculinizada donde todos los servicios están organizados para satisfacer las necesidades de los hombres, especialmente vistos como personas no calificadas movidas por el interés monetario, los "rotos con plata", y cada vez menos chilenos a medida que aumenta la distancia. Los hombres evalúan de manera más ambivalente la región, al percibir más positivamente las oportunidades laborales y de ingresos que ofrece, aunque mantienen una visión negativa sobre la calidad de vida que pueden encontrar en sus ciudades y un discurso crítico sobre la actividad de las grandes empresas mineras. Estos discursos ayudan a explicar la baja atracción de mujeres migrantes a la Región de Antofagasta y la casi nula participación de las mujeres en la conmutación hacia Antofagasta, por considerarse una opción exclusivamente masculina.

El discurso de los "rotos con plata", que supone una proletarización, acompañada de desprecio por aquellos que deciden ir a vivir a la Región de Antofagasta, se encuentra con fuerza en Coquimbo y Santiago, especialmente en la primera y entre las mujeres, y se transforma en el discurso del "colonizador" al llegar a Valdivia. Es difícil determinar en qué medida este tipo de discurso pueda llegar a inhibir flujos de migrantes profesionales hacia Antofagasta. Lo cierto es que la composición de estos flujos difiere significativamente según la región considerada. En las regiones de Coquimbo y Los Ríos predomina la emisión de migrantes hacia Antofagasta con educación media y funciones de operarios y personal no calificado. En contraste, la Región Metropolitana se encuentra especializada en enviar hacia Antofagasta migrantes con mayor nivel educativo y funciones técnicas y profesionales.

El conjunto de discursos encontrados sobre la Región de Antofagasta contribuye a conformar un espacio cuya representación se caracteriza por la existencia de ciudades sin atractivo para vivir, si bien con oportunidades para lucrar gracias a la minería. El análisis complementario de los flujos de migración y conmutación hacia 
esta región refleja que estos discursos podrían estar afectando de manera negativa la atracción de trabajadores calificados, lo cual se ve intensificado por la oportunidad de conmutar que ofrece la propia minería. De esta manera, el carácter no sustentable de la actividad minera se transfiere a la representación de la región y se refuerza al limitar la atracción de trabajadores que, por sus características, podrían contribuir a aumentar su diversificación productiva.

Estos discursos tienen implicaciones directas en el diseño de las políticas orientadas a promover el desarrollo de la Región de Antofagasta. Muestran, en primer lugar, la conveniencia de diferenciar entre las acciones según su escala espacial regional o urbana. En el ámbito regional es difícil tomar medidas precisas, dado que las representaciones de Antofagasta no coinciden con regiones administrativas. En este caso, las acciones tendrían un carácter más general, orientado a lo que en los discursos se denomina como el "Norte" o las "zonas extremas". Desde una perspectiva simbólica, podrían acometerse acciones de carácter nacional basadas en la revalorización del desierto y la minería, así como orientadas al reconocimiento de una identidad chilena diversa, a partir de políticas educativas y mediáticas que reconocieran la chilenidad de las zonas extremas del país. En el ámbito urbano es posible realizar medidas más concretas, orientadas a la reformulación positiva del discurso sobre las "ciudades mineras". Entre ellas existirían acciones de carácter material, que buscarían la dotación de infraestructura, condiciones de seguridad y trabajo orientadas a aumentar el atractivo de estas ciudades como lugar de vida. Estas inversiones materiales, sin embargo, tendrían un efecto limitado si no se abordan los aspectos simbólicos que provocan el rechazo a vivir en estas ciudades, como los discursos sobre las "ciudades campamento" y los "rotos con plata". En cualquier caso, se debe tener en cuenta el carácter sociohistórico de los discursos y, por lo tanto, la dificultad de modificarlos y obtener resultados a corto plazo.

\section{Referencias bibliográficas}

Alonso, L. E. (1998). La mirada cualitativa en sociología. Madrid: Fundamentos.

Aroca, P. \& Atienza, M. (2008). La conmutación regional en Chile y su impacto en la Región de Antofagasta. EURE, 34(102), 97-121. http://dx.doi.org/10.4067/S025071612008000200006

Aroca, P. \& Atienza, M. (2011). Economic implications of long distance commuting in the Chilean mining industry. Resources Policy, 36(3), 196-203. Recuperado de http://ideas. repec.org/p/cat/dtecon/dt201003.html

Bailly, A. (1989). Lo imaginario espacial y la geografía: en defensa de la geografía de las representaciones. Anales de Geografía de la Universidad Complutense, 9, 11-19. Recuperado de http://revistas.ucm.es/index.php/AGUC/article/view/AGUC8989110011A

Callejo, J. (2001). El grupo de discusión. Introducción a una práctica de investigación. Barcelona: Ariel. 
Canales, M. \& Peinado, A. (1994). Grupo de discusión. En J. Gutiérrez \& J. M. Delgado (Eds.), Métodos y técnicas cualitativas de investigación social (pp. 288-311). Madrid: Síntesis.

Colectivo IOÉ (2010).¿Para quésirve el grupo de discusión? Una revisión crítica del uso de técnicas grupales en los estudios sobre migraciones. Empiria, 19, 73-99. Recuperado de http:// www.colectivoioe.org/uploads/d70e92469ea5d534c31d5b25acaf48ae0c593b25.pdf

Comisión Chilena del Cobre (Cochilco) (varios años). Estadísticas. En http://www.cochilco.cl/ estadisticas/intro-bd.asp

Comisión Económica para América Latina y el Caribe (CEPAL). (2009). Economía y territorio en América Latina y el Caribe: desigualdades y politicas. Libros de la CEPAL 99. Santiago de Chile: Autor. Recuperado de http://www.eclac.org/publicaciones/xml/9/36309/ LCG2385.pdf

Conde Gutiérrez del Álamo, F. (2009). Análisis sociológico del sistema de discursos. Cuadernos Metodológicos n. ${ }^{\circ} 33$ (1ª reimpr., 2010). Madrid: Centro de Investigaciones Sociológicas (CIs).

Fuller G. \& Chapman, M. (1974). On the role of mental maps in migration research. International Migration Review, 8(4), 491-506.

Gould, P. \& White, R. (1974). Mental maps. Harmondsworth: Penguin Books.

Gutiérrez Brito, J. (2008). Dinámica del grupo de discusión. Cuadernos Metodológicos, n. ${ }^{\circ} 41$. Madrid: Centro de Investigaciones Sociológicas (CIs).

Hiernaux, D. (2007). Los imaginarios urbanos: de la teoría y los aterrizajes en los estudios urbanos. EURE, 33(99), 17-30. http://dx.doi.org/10.4067/S0250-71612007000200003

Ibánez, J. (1979). Más allá de la sociología. El grupo de discusión: técnica y crítica. Madrid: Siglo Xxi.

Instituto Nacional de Estadísticas (INE), Chile. (2002). XVII Censo Nacional de Población y VI de Vivienda. En http://www.ine.cl/cd2002/

Lindón, A. (2007). Los imaginarios urbanos y el constructivismo geográfico: los hologramas espaciales. EURE, 33(99), 31-46. http://dx.doi.org/10.4067/S025071612007000200004

Lowenthal, D. (1961). Geography, experience and imagination: towards a geographical epistemology. Annals of the Association of American Geographers. 51(3), 241-260. doi: 10.1111/j.1467-8306.1961.tb00377.x

Lynch, K. (1960). The Image of the City. Cambridge: The.MIT Press

Massey, D. (1994). Space, place, and gender. Minneapolis: University of Minnesota Press.

Massey, D. (2005). For space. London: Sage.

Ministerio de Planificación, Chile. (2009). Encuesta CASEN 2009. La Encuesta de Caracterización Socioeconómica Nacional [Publicación Base de Datos: 5 de agosto 2010). Disponible en http://www.ministeriodesarrollosocial.gob.cl/casen2009/

Organización para la Cooperación y el Desarrollo Económicos (OCDE). (2009). Estudios territoriales de la OCDE: Chile. Santiago de Chile: OECD / Minsterio del Interior, Chile. Recuperado de http://www.ministeriodesarrollosocial.gob.cl/btca/txtcompleto/est. ocdeCh.pdf

Ortí, A. (1989). La apertura y el enfoque cualitativo o estructural: la entrevista abierta y la discusión de grupo. En M. García, J- Ibáñez \& F. Alvira, El análisis de la realidad social. Métodos y técnicas de investigación (pp. 189-221). Madrid: Alianza. 
Perroux, F. (1950). Economic space: Theory and applications. The Quarterly Journal of Economics, 64(1), 89-104. doi: 10.2307/1881960

Rodrigo, L. M. (2011). Discursos sobre la Región de Antofagasta en el contexto de la migración regional. Antofagasta: Ediciones Universidad Católica del Norte, Observatorio Regional de Desarrollo Humano (ordHum).

Storper, M. \& Scott A. J. (2009). Rethinking human capital, creativity and urban growth. Journal of Economic Geography, 9(2), 147-167. doi: 10.1093/jeg/lbn052

Vara Muñoz, J. L. (2008). Cinco décadas de Geografía de la percepción. Ería, 77, 371-384. Recuperado de dialnet.unirioja.es/descarga/articulo/2927235.pdf. 